\title{
Distortion Type Theorems for Functions in the Logarithmic Bloch Space
}

\author{
Armando J. García-Ortíz, ${ }^{1}$ Milton del Castillo Lesmes Acosta, ${ }^{2}$ \\ and Julio C. Ramos-Fernández ${ }^{3}$ \\ ${ }^{1}$ Área de Matemática, Universidad Nacional Experimental de Guayana, Pto. Ordaz, Bolívar, Venezuela \\ ${ }^{2}$ Proyecto Curricular de Matemáticas, Facultad de Ciencias y Educación, Universidad Distrital Francisco José de Caldas, \\ Carrera 3 No. 26 A-40, Bogotá, Colombia \\ ${ }^{3}$ Departamento de Matemática, Universidad de Oriente, 6101 Cumaná, Sucre, Venezuela
}

Correspondence should be addressed to Julio C. Ramos-Fernández; jcramos@udo.edu.ve

Received 23 January 2017; Revised 22 March 2017; Accepted 2 April 2017; Published 16 April 2017

Academic Editor: John R. Akeroyd

Copyright (C) 2017 Armando J. García-Ortíz et al. This is an open access article distributed under the Creative Commons Attribution License, which permits unrestricted use, distribution, and reproduction in any medium, provided the original work is properly cited.

We establish distortion type theorems for locally schlicht functions and for functions having branch points satisfying a normalized Bloch condition in the closed unit ball of the logarithmic Bloch space $\mathscr{B}_{\log }$. As a consequence of our results we have estimations of the schlicht radius for functions in these classes.

\section{Introduction}

One of the most important results in the area of geometric theory of functions of a complex variable is the celebrated distortion's theorem established by Koebe and Bieberbach $[1,2]$ at the beginning of the twentieth century. Koebe and Bieberbach showed that the range of any function $f$ in the class $\delta$ of all conformal functions on $\mathbb{D}$, the open unit disk of the complex plane $\mathbb{C}$, normalized such that $f(0)=0=$ $f^{\prime}(0)-1$ contain the Euclidean disk with center at the origin and radius $1 / 4$. This last result is today known as Koebe $1 / 4$ Theorem and, in particular, shows that Bloch's constant (see [3]) is greater than or equal to $1 / 4$. Koebe and Bieberbach found sharp lower and upper bounds for the growth and the distortion of conformal maps in the class $\mathcal{S}$; more precisely, they showed that for any $f \in \mathcal{S}$ and $z \in \mathbb{D}$ the following estimations hold.

(1) Growth theorem:

$$
\frac{|z|}{(1+|z|)^{2}} \leq|f(z)| \leq \frac{|z|}{(1-|z|)^{2}}
$$

(2) Distortion theorem:

$$
\frac{1-|z|}{(1+|z|)^{3}} \leq\left|f^{\prime}(z)\right| \leq \frac{1+|z|}{(1-|z|)^{3}}
$$

with equality if and only if $f$ is a rotation of the Koebe function defined by

$$
K(z)=\frac{z}{(1-z)^{2}}, \quad(z \in \mathbb{D}),
$$

which also belongs to the class $\mathcal{S}$. In particular, the distortion theorem implies that the class $\mathcal{S}$ is contained in the closed ball with center at the origin and radius 8 of $p$-Bloch space $\mathscr{B}_{p}$ for all $p \geq 3$ (see Section 3 for the definition of $\mathscr{B}_{p}$ ). For more properties of conformal maps and distortion theorem, we recommend the excellent books $[4,5]$.

Although the distortion theorem gives sharp bounds for the modulus of the derivative of functions in the class $\delta$, it cannot be applied to the bigger class of locally schlicht functions defined on $\mathbb{D}$ satisfying the normalized Bloch conditions $f(0)=0=f^{\prime}(0)-1$ (recall that a holomorphic function $f$ is locally schlicht on $\mathbb{D}$ if $f^{\prime}(z) \neq 0$ for all $z \in$ $\mathbb{D})$. Many authors have obtained distortion type theorems or lower bounds for the modulus or real part of the derivative of locally schlicht functions in Bloch-type spaces. The pioneer work about this subject appears in 1992 and is due to Liu and Minda [6]. They established distortion theorems for locally schlicht functions $f$ in the classical Bloch space $\mathscr{B}$ satisfying the conditions $f(0)=0, f^{\prime}(0)=1$, and $\|f\|_{\mathscr{B}}=1$ (see Section 3 for the definition of Bloch space). Liu and Minda 
give sharp lower bounds for $\left|f^{\prime}(z)\right|$ and for $\operatorname{Re} f^{\prime}(z)$ and as consequence of their results they obtain a lower bound for Bloch's constant. Determination of the (locally schlicht) Bloch constant is still an open problem. By Landau's reduction, it is enough to consider those functions with Bloch seminorm not greater than 1 . Hence, it is important to consider certain subclasses of functions in Bloch spaces having seminorm not greater than 1 .

The results of Liu and Minda in [6] have been extended to other classes of locally schlicht functions or to functions having branch points in the Bloch space by Yanagihara [7], Bonk et al. [8, 9], and Graham and Minda [10]. The extension of the above results to $p$-Bloch spaces was obtained by Terada and Yanagihara [11] and by Zheng and Wang [12]. It is an open problem to obtain distortion type theorems for locally schlicht functions in other spaces of analytic functions.

In this article we extend the results of Liu and Minda [6] to the logarithmic Bloch space $\mathscr{B}_{\log }$ which we define in Section 3; we obtain lower bounds for the modulus and the real part of the derivative of locally schlicht functions and for functions having branch points in the closed unit ball of $\mathscr{B}_{\log }$ satisfying a normalized Bloch condition $f(0)=0=f^{\prime}(0)-1$. Our results will be showed in Sections 4 and 5 , as consequence of our results, in Section 6, we obtain lower bounds for the schlicht radius of functions in these classes.

\section{Some Preliminaries: Julia's Lemma}

In this section we gather some notations, definitions, and results that we will need through this note. We denote by $\mathbb{D}$ the open unit disk in the complex plane $\mathbb{C}$, with center at the origin and radius $1 ; \partial \mathbb{D}$ denotes the boundary of $\mathbb{D}$. The space of all complex and holomorphic functions on $\mathbb{D}$, as is usual, is denoted by $H(\mathbb{D})$. A function $f \in H(\mathbb{D})$ is said to be normalized if $f(0)=0$ and $f^{\prime}(0)=1$ and $f$ is locally schlicht or locally univalent if $f^{\prime}(z) \neq 0$ for all $z \in \mathbb{D}$. A point $z_{0}$ is a branch point for $f$ if $f^{\prime}\left(z_{0}\right)=0$. For $r>0$, we define

$$
\Delta(1, r)=\left\{z \in \mathbb{D}: \frac{|1-z|^{2}}{1-|z|^{2}}<r\right\}
$$

$\Delta(1, r)$ is known as a horodisk $\mathbb{D}$; that is, it is an Euclidean disk contained in $\mathbb{D}$ which is tangent to $\partial \mathbb{D}$ at 1 . Furthermore, $\Delta(1, r)$ has center at $1 /(1+r)$ and radius $r /(1+r)$. The closure of $\Delta(1, r)$ relative to $\mathbb{D}$ is denoted by $\bar{\Delta}(1, r)$. Observe that $1 \notin$ $\bar{\Delta}(1, r)$ but $(1-r) /(1+r) \in \bar{\Delta}(1, r)$. With these notations, we can enunciate the well known Julia's Lemma; the reader can consult the excellent book of Ahlfors [13] for its proof.

Lemma 1 (Julia's Lemma). Suppose that $w$ is a complex and holomorphic function on $\mathbb{D} \cup\{1\}$ such that $w$ maps $\mathbb{D}$ into $\mathbb{W}^{+}=$ $\{z \in \mathbb{C}: \operatorname{Re}(z)>0\}$, the right half-plane, and $w(1)=0$. Then, for any $r>0$, the function $w$ maps the horodisk $\bar{\Delta}(1, r)$ into the Euclidean disk $\{z \in \mathbb{C}:|z-d r|<d r\}$, where $d=-w^{\prime}(1)>0$. Furthermore, a boundary point of the first disk is mapped on the boundary of the second disk if and only if $w$ is a conformal function mapping $\mathbb{D}$ onto $\mathbb{U}^{+}$and satisfying $w(1)=0$.
In 1992, Liu and Minda [6] established distortion theorem for functions in the Bloch space; they showed the following results which are consequences of Julia's Lemma. We include the proof of the first one to illustrate the application of Julia's Lemma.

Lemma 2 ([6, corollary in Section 1]). Let w be a holomorphic function on $\mathbb{D} \cup\{1\}$. Suppose that $w$ maps $\mathbb{D}$ into the right halfplane $\mathbb{U}^{+}$and that $w(1)=0$. Then $d=-w^{\prime}(1)>0$ and

$$
\operatorname{Re} w(x) \leq 2 d \frac{1-x}{1+x},
$$

for all $x \in(-1,1)$, with equality for some $x \in(-1,1)$ if and only if

$$
w(z)=2 d \frac{1-z}{1+z},
$$

for all $z \in \mathbb{D}$.

Proof. Indeed, let us fix $x \in(-1,1)$; then $r=(1-x) /(1+x)>$ 0 and by Julia's Lemma, $w$ maps $\bar{\Delta}(1, r)$ into the Euclidean disk $D(d r, d r)$. In particular, since $x \in \bar{\Delta}(1, r)$, then $w(x) \in$ $D(d r, d r)$; this fact implies that $\operatorname{Re} w(x) \leq 2 d r$. Furthermore, $x \in \partial \Delta(1, r)$; hence if $\operatorname{Re} w(x)=2 d r$, then we conclude that $w(x)=2 d r \in \partial D(d r, d r)$ and, by Julia's Lemma, this last fact occurs if $w$ is the conformal map from $\mathbb{D}$ onto $\mathbb{U}^{+}$such that $w(1)=0$; that is, $w(z)=2 d((1-z) /(1+z))$ for all $z \in \mathbb{D}$. This shows the lemma.

Lemma 3 ([6, corollary to Theorem 3$])$. Let $f$ be a holomorphic function on $\mathbb{D} \cup\{1\}$. Suppose that $f(\mathbb{D}) \subset \mathbb{D}, f(1)=1$ and that all the zeros of $f$ have multiplicity at least $n$. If $f^{\prime}(1)=n$, then

(1) $|f(x)| \geq x^{n}$ for all $x \in[0,1)$, with equality for some $x \in[0,1)$ if and only if $f(z)=z^{n}$ for all $z \in \mathbb{D}$;

(2) $\operatorname{Re}(f(x)) \geq x^{n}$ for all $(n-1) /(n+1) \leq x<1$, with equality for some $x \in[(n-1) /(n+1), 1)$ if and only if $f(z)=z^{n}$ for all $z \in \mathbb{D}$.

We finish this section by establishing the following elementary property of the complex exponential. We thank the reviewer for providing us the following simple demonstration of this fact.

Lemma 4. Let $x \in[0,1)$ be fixed and $D_{x}$ the Euclidean disk with center at $(1-x) /(1+x)$ and radius $(1-x) /(1+x)$; then

$$
\min \left\{\operatorname{Re}(\exp (-z)): z \in D_{x}\right\}=\exp \left(-2 \frac{1-x}{1+x}\right) .
$$

Proof. Let $r=(1-x) /(1+x)$ for simplicity and let $f(z)=$ $e^{r(z-1)}$. Since $1+z f^{\prime \prime}(z) / f^{\prime}(z)=1-r z$ has positive real part on $|z|<1$, the function $f$ is convex. In particular, $\operatorname{Re}(f(z))>$ $f(-1)=e^{-2 r}$, which proves the assertion.

\section{Logarithmic Bloch Space}

In this section we gather the definition and some of the properties of the logarithmic Bloch space $\mathscr{B}_{\log }$. Let us recall 
that a function weight $\mu$ on $\mathbb{D}$ is a bounded, positive, and continuous function defined on $\mathbb{D}$. Given a weight $\mu$ on $\mathbb{D}$, $\mu$-Bloch space, denoted by $\mathscr{B}_{\mu}$, consists of all holomorphic functions $f$ on $\mathbb{D}$ such that

$$
\|f\|_{\mu}:=\sup _{z \in \mathbb{D}} \mu(z)\left|f^{\prime}(z)\right|<\infty .
$$

It is known that if the weight $\mu$ is radial, that is, $\mu(z)=\mu(|z|)$ for all $z \in \mathbb{D}$, then $\mathscr{B}_{\mu}$ is a Banach space with the norm $\|f\|_{\mathscr{B}_{\mu}}=|f(0)|+\|f\|_{\mu}$. When $\mu(z)=1-|z|^{2}$, with $z \in \mathbb{D}, \mathscr{B}_{\mu}$ becomes the Bloch space which is denoted by $\mathscr{B}$, while when $\mu(z)=\left(1-|z|^{2}\right)^{p}$, with $z \in \mathbb{D}$ and $p>0$ fixed, we obtain $p$-Bloch space which is denoted by $\mathscr{B}_{p}$.

Clearly, the function $\mu_{\log }$, defined by

$$
\mu_{\log }(z)=\left[\log \left(\frac{e}{1-|z|^{2}}\right)\right]^{-1},
$$

defines a weight on $\mathbb{D}$. Hence, the space $\mathscr{B}_{\log }=\mathscr{B}_{\mu_{\log }}$ is a Banach space with the norm

$$
\begin{aligned}
\|f\|_{\mathscr{B}_{\log }} & =|f(0)|+\|f\|_{\log } \\
& :=|f(0)|+\sup _{z \in \mathbb{D}} \frac{\left|f^{\prime}(z)\right|}{\log \left(e /\left(1-|z|^{2}\right)\right)} .
\end{aligned}
$$

We call $\mathscr{B}_{\log }$ as the logarithmic Bloch space. In the next result we are going to show that $\mathscr{B}_{\log }$ is a subspace of $\mathscr{B}_{p}$ for all $p \geq 1$.

Proposition 5. The space $\mathscr{B}_{\log }$ is contained in $\mathscr{B}_{p}$, for all $p \geq$ 1. Furthermore,

$$
\|f\|_{\mathscr{B}_{p}} \leq\|f\|_{\mathscr{B}_{\log }},
$$

for all function $f \in \mathscr{B}_{\log }$.

Proof. It is enough to show that for $p \geq 1$ fixed

$$
\left(1-|z|^{2}\right)^{p} \log \left(\frac{e}{1-|z|^{2}}\right) \leq 1,
$$

for all $z \in \mathbb{D}$. But, this last inequality is true since the function

$$
h(t)=t^{p} \log \left(\frac{e}{t}\right)-1
$$

with $t \in(0,1]$, is increasing and $h(1)=0$.

Also, we have the following very useful identity (see Lemma 3.3 in [12]).

Lemma 6. If $f \in \mathscr{B}_{\log }, f(0)=0, f^{\prime}(0)=1$, and $\|f\|_{\log } \leq 1$, then $f^{\prime \prime}(0)=0$.

Proof. Suppose that $f \in \mathscr{B}_{\text {log }}, f(0)=0, f^{\prime}(0)=1$, and $\|f\|_{\log } \leq 1$. Then, for each $z \in \mathbb{D}$, we have

$$
\left|f^{\prime}(z)\right| \leq \log \left(\frac{e}{1-|z|^{2}}\right)
$$

Taylor's theorem implies that

$$
\begin{aligned}
\mid 1 & +f^{\prime \prime}(0) z+\left.o(|z|)\right|^{2} \leq \log ^{2}\left(\frac{e}{1-|z|^{2}}\right) \\
& =\left(1+|z|^{2}+o(|z|)\right)^{2}
\end{aligned}
$$

as $z \rightarrow 0$. But since

$$
\begin{aligned}
\mid 1 & +f^{\prime \prime}(0) z+\left.o(|z|)\right|^{2} \\
& =1+2 \operatorname{Re}\left(f^{\prime \prime}(0) z\right)+o(|z|),
\end{aligned}
$$

as $z \rightarrow 0$, and

$$
\left(1+|z|^{2}+o(|z|)\right)^{2}=1+o(|z|),
$$

as $z \rightarrow 0$, we obtain from (15) that

$$
2 \operatorname{Re}\left(f^{\prime \prime}(0) z\right) \leq o(|z|)
$$

as $z \rightarrow 0$. Now, if we consider $z=r \overline{f^{\prime \prime}(0)} /\left|f^{\prime \prime}(0)\right|$ with $r>0$ small in (18), we conclude $\left|f^{\prime \prime}(0)\right|=0$ and we are done.

The following functions play a very important role in our work; they will be used to get lower bounds for locally schlicht functions and for functions having branch points in certain classes in the logarithmic Bloch space. From now, we use $\log (w)$ to denote the principal logarithmic of the complex number $w \neq 0$. Observe that the principal logarithmic is a holomorphic function on $D(1,1)$, the Euclidean disk with center at 1 and radius 1 :

(1) For each $n \in \mathbb{N}$, we set

$$
F_{n}(z)=\int_{0}^{z}\left(\frac{1-s / a_{n}}{1-a_{n} s}\right)^{n}\left(1-2 \log \left(1-a_{n} s\right)\right) d s,
$$

where $a_{n}=\sqrt{n /(n+2)}$ and $z \in \mathbb{D}$. Clearly, $F_{n} \in H(\mathbb{D})$ for all $n \in \mathbb{N}, F_{n}(0)=0$, and $F_{n}^{\prime}(0)=1$.

(2) For $z \in \mathbb{D}$, we define

$$
F(z)=\int_{0}^{z} \exp \left(-\frac{2 s}{1-s}\right)(1-2 \log (1-s)) d s .
$$

We can see that $F \in H(\mathbb{D}), F(0)=0$, and $F^{\prime}(0)=1$.

Also we have that the function $F$ satisfies the following properties.

Proposition 7. The function $F$ belongs to $\mathscr{B}_{\log }$. Furthermore, $\sup _{z \in \mathbb{D}} \mu_{\log }(z)\left|F^{\prime}(|z|)\right|=1$ but $\|F\|_{\log }>1$.

Proof. We see that $\|F\|_{\log }>1$. Indeed, we have

$$
\begin{aligned}
& \|F\|_{\log } \geq \frac{\left|F^{\prime}(i / 2)\right|}{1-\log \left(1-|i / 2|^{2}\right)} \\
& \quad=\frac{\exp (2 / 5)}{1-\log (3 / 4)} \sqrt{\left(1-\log \left(\frac{5}{4}\right)\right)^{2}+4 \arctan ^{2}\left(\frac{1}{2}\right)} \\
& \quad \approx 1.4014837>1 .
\end{aligned}
$$


Now, we are going to show that $F \in \mathscr{B}_{\log }$. Since the function $\exp (-2 z /(1-z))$ is holomorphic on $\mathbb{D}$, then the modulus maximum principle tells us that its maximum value is attained in the boundary $\partial \mathbb{D}$. But if $|z|=1$ then $|1-z|^{2}=$ $2(1-\operatorname{Re}(z))$ and hence

$$
\begin{aligned}
\sup _{z \in \mathbb{D}}\left|\exp \left(-\frac{2 z}{1-z}\right)\right| & =\sup _{|z|=1} \exp \left(2-\frac{2(1-\operatorname{Re}(z))}{|1-z|^{2}}\right) \\
& =e .
\end{aligned}
$$

On the other hand, for each $z \in \mathbb{D}$, we have $|\arg (1-z)|<\pi / 2$ and

$$
\sup _{z \in \mathbb{D}} \frac{|\arg (1-z)|}{1-\log \left(1-|z|^{2}\right)} \leq \frac{\pi}{2}
$$

Furthermore, using elementary calculus, we can see that the real function $H(t)=e^{2}-(1-t)(1+t)^{3}$ is nonnegative for all $t \in[0,1]$ (its minimum value is $H(1 / 2)=e^{2}-27 / 16 \approx 5.70$ ). Hence for any $t \in[0,1)$ we obtain

$$
\begin{aligned}
1- & 2 \log (1-t)-3\left(1-\log \left(1-t^{2}\right)\right) \\
= & \log \left(\frac{(1-t)(1+t)^{3}}{e^{2}}\right) \leq 0 .
\end{aligned}
$$

This last implies that

$$
\frac{1-2 \log (1-t)}{1-\log \left(1-t^{2}\right)} \leq 3
$$

for all $t \in[0,1)$. We conclude that for any $z \in \mathbb{D}$ such that $|1-z|^{2} \leq e$

$$
\begin{aligned}
\frac{\left|\log \left(e /|1-z|^{2}\right)\right|}{1-\log \left(1-|z|^{2}\right)} & =\frac{1-2 \log (|1-z|)}{1-\log \left(1-|z|^{2}\right)} \\
& \leq \frac{1-2 \log (1-|z|)}{1-\log \left(1-|z|^{2}\right)} \leq 3,
\end{aligned}
$$

while for $z \in \mathbb{D}$ such that $|1-z|^{2}>e$ we have

$$
\frac{\left|\log \left(e /|1-z|^{2}\right)\right|}{1-\log \left(1-|z|^{2}\right)}=\frac{\log \left(|1-z|^{2} / e\right)}{1-\log \left(1-|z|^{2}\right)} \leq \log (4)-1
$$

These last inequalities, (22) and (23), imply that

$$
\begin{aligned}
\|F\|_{\log } & \leq e\left(3+\log (4)-1+2\left(\frac{\pi}{2}\right)\right) \\
& =e(2+\log (4)+\pi)
\end{aligned}
$$

which shows that $F \in \mathscr{B}_{\log }$.

Now, we are going to show that $\sup _{z \in \mathbb{D}} \mu_{\text {log }}(z)\left|F^{\prime}(|z|)\right|=1$. Observe that $\mu_{\log }(0)\left|F^{\prime}(0)\right|=1$. Also the real function $H(t)=$ $\exp (-2 t /(1-t))(1-2 \log (1-t))-1+\log \left(1-t^{2}\right)$ with $t \in[0,1)$ satisfies $H(0)=0, H(t) \rightarrow-\infty$ as $t \rightarrow 1^{-}$and it is strictly decreasing since

$$
\begin{aligned}
H^{\prime}(t) \\
=-\frac{2 t}{1-t^{2}} \\
\quad-\frac{2}{1-t}\left(\frac{2}{1-t} \log \left(\frac{e}{(1-t)^{2}}\right)-1\right) \exp \left(-\frac{2 t}{1-t}\right)
\end{aligned}
$$

$<0$,

for all $t \in[0,1)$. Hence we conclude that $H(t) \leq 0$ for all $t \in[0,1)$ which shows the affirmation.

For the sequence $\left\{F_{n}\right\}$, we have the following properties.

Proposition 8. Functions $F_{n}$ with $n \in \mathbb{N}$ belong to $\mathscr{B}_{\log }$ and satisfy

$$
\lim _{n \rightarrow \infty} F_{n}^{\prime}(z)=F^{\prime}(z),
$$

for each $z \in \mathbb{D}$. Furthermore, for each $n \in \mathbb{N}\left\|F_{n}\right\|_{\log }>1$, in fact, $\sup _{z \in \mathbb{D}} \mu_{\log }(z)\left|F_{n}^{\prime}(|z|)\right|>1$.

Proof. Clearly, for any $n \in \mathbb{N}$, the function $F_{n}$ belongs to $\mathscr{B}_{\log }$ since $F_{n} \in H(\overline{\mathbb{D}})$. We are going to show that $\sup _{z \in \mathbb{D}} \mu_{\log }(z)\left|F_{n}^{\prime}(|z|)\right|>1$. It is enough to show that there exists a $t_{0} \in\left(a_{n}, 1\right)$ such that $H\left(t_{0}\right)>0$, where

$$
\begin{aligned}
H(t)= & \left(\frac{t / a_{n}-1}{1-a_{n} t}\right)^{n} \log \left(\frac{e}{\left(1-a_{n} t\right)^{2}}\right) \\
& -\log \left(\frac{e}{1-t^{2}}\right)
\end{aligned}
$$

with $t \in\left(a_{n}, 1\right)$. Observe that, for $t_{n}=r a_{n}$ with $r=2 /(1+$ $\left.a_{n}^{2}\right)>1$, we have $t_{n} \in\left(a_{n}, 1\right), r-1=1-r a_{n}^{2}$, and $1-r^{2} a_{n}^{2}=$ $\left(1-r a_{n}^{2}\right)^{2}$ which implies that $H\left(t_{n}\right)=0$. Also,

$$
\begin{aligned}
& H^{\prime}(t) \\
& =n\left(\frac{t / a_{n}-1}{1-a_{n} t}\right)^{n-1} \frac{1 / a_{n}-a_{n}}{\left(1-a_{n} t\right)^{2}} \log \left(\frac{e}{\left(1-a_{n} t\right)^{2}}\right) \\
& \quad+\left(\frac{t / a_{n}-1}{1-a_{n} t}\right)^{n} \frac{2 a_{n}}{1-a_{n} t}-\frac{2 t}{1-t^{2}} ;
\end{aligned}
$$

hence

$$
\begin{gathered}
H^{\prime}\left(t_{n}\right)=n \frac{1 / a_{n}-a_{n}}{\left(1-r a_{n}^{2}\right)^{2}} \log \left(\frac{e}{\left(1-r a_{n}^{2}\right)^{2}}\right)+\frac{2 a_{n}}{1-r a_{n}^{2}} \\
-\frac{2 r a_{n}}{1-r^{2} a_{n}^{2}}=\frac{n}{\left(1-r a_{n}^{2}\right)^{2}}\left(\frac{1}{a_{n}}-a_{n}\right) \\
\cdot\left(\log \left(\frac{e}{\left(1-r a_{n}^{2}\right)^{2}}\right)-\frac{1}{n} \frac{2 a_{n}^{2}}{1-a_{n}^{2}}\right)
\end{gathered}
$$




$$
\begin{aligned}
& =\frac{n}{\left(1-r a_{n}^{2}\right)^{2}}\left(\frac{1}{a_{n}}-a_{n}\right) \\
& \cdot\left(\log \left(\frac{e}{\left(1-r a_{n}^{2}\right)^{2}}\right)-1\right),
\end{aligned}
$$

since $r-1=1-r a_{n}^{2}, 1-r^{2} a_{n}^{2}=\left(1-r a_{n}^{2}\right)^{2}$, and $r\left(a_{n}^{2}+1\right)=2$ and we have used that $a_{n}=\sqrt{n /(n+2)}$ in the last equality. Thus, we conclude that $H^{\prime}\left(t_{n}\right)>0$ and since $H\left(t_{n}\right)=0$, then there exists $t_{0} \in\left(t_{n}, 1\right)$ such that $H\left(t_{0}\right)>0$. This shows the affirmation. The other properties of $F_{n}$ 's are clear.

\section{A Distortion Theorem for Locally Schlicht Functions in $\mathscr{B}_{\log }$}

In this section we establish a distortion theorem for locally schlicht functions in the closed unit ball of $\mathscr{B}_{\log }$ satisfying normalized Bloch conditions. We denote by $\beta_{\log }^{(\infty)}$ the class of all holomorphic functions $f \in \mathscr{B}_{\log }$ such that $f$ is locally schlicht, $f(0)=0, f^{\prime}(0)=1$, and $\|f\|_{\log } \leq 1$. With these notations, we have the following result.

Theorem 9. If $f \in \beta_{\log }^{(\infty)}$ then we have the following:

(1) $\left|f^{\prime}(z)\right| \geq F^{\prime}(|z|)=\exp (-2|z| /(1-|z|))(1-2 \log (1-$ $|z|))$ for all $z \in \mathbb{D}$. There is not a function $f_{0} \in \beta_{\log }^{(\infty)}$ such that $\left|f_{0}^{\prime}\left(z_{0}\right)\right|=F^{\prime}\left(\left|z_{0}\right|\right)$ for some $z_{0} \in \mathbb{D} \backslash\{0\}$.

(2) $\operatorname{Re} f^{\prime}(z) \geq F^{\prime}(|z|)=\exp (-2|z| /(1-|z|))(1-2 \log (1-$ $|z|)$ ) for all $|z| \leq 1 / 2$. There is not a function $f_{0} \in \beta_{\log }^{(\infty)}$ such that $\operatorname{Re} f_{0}^{\prime}\left(z_{0}\right)=F^{\prime}\left(\left|z_{0}\right|\right)$ for some $z_{0} \in D(0,1 / 2) \backslash$ $\{0\}$.

Proof. (1) Suppose that $f \in \beta_{\log }^{(\infty)}$. Let us fix $|\zeta|=1$ and we set the function

$$
g(u)=\left(1-2 \log \left(\frac{1+u}{2}\right)\right)^{-1} f^{\prime}\left(\frac{1-u}{2} \zeta\right)
$$

with $u \in \mathbb{D}$, where $\log (w)$ denotes the principal logarithmic of $w \in D(1,1)$. Clearly $g$ is holomorphic on $\overline{\mathbb{D}} \backslash\{-1\}$ and $g(1)=1$ because $f^{\prime}(0)=1$. Since $f$ is locally schlicht on $\mathbb{D}$, we have that $g(z) \neq 0$ for all $z \in \mathbb{D}$. Furthermore,

$$
\begin{aligned}
& g^{\prime}(u) \\
& =\frac{2}{1+u}\left(1-2 \log \left(\frac{1+u}{2}\right)\right)^{-2} f^{\prime}\left(\frac{1-u}{2} \zeta\right) \\
& \quad+\left(1-2 \log \left(\frac{1+u}{2}\right)\right)^{-1} f^{\prime \prime}\left(\frac{1-u}{2} \zeta\right)\left(-\frac{\zeta}{2}\right) .
\end{aligned}
$$

In particular, $g^{\prime}(1)=1$ since $f^{\prime}(0)=1$ and $f^{\prime \prime}(0)=0$ (by Lemma 6). Also, for any $u \in \mathbb{D}$, we have

$$
\begin{aligned}
& |g(u)|=\left|1-2 \log \left(\frac{1+u}{2}\right)\right|^{-1}\left|f^{\prime}\left(\frac{1-u}{2} \zeta\right)\right| \\
& \leq \frac{1}{|\operatorname{Re}(1-2 \log ((1+u) / 2))|} \\
& \cdot \log \left(\frac{e}{1-|(1-u) / 2|^{2}}\right)=\frac{1}{\log \left(e /|(1+u) / 2|^{2}\right)} \\
& \cdot \log \left(e / 1-|(1-u) / 2|^{2}\right)<1
\end{aligned}
$$

and $g$ maps $\mathbb{D}$ into $\mathbb{D} \backslash\{0\}$. Hence, there exists a holomorphic function $w$ mapping the unit disk $\mathbb{D}$ into the right half-plane $\mathbb{M}^{+}=\{z: \operatorname{Re}(z)>0\}$ and such that $g(u)=\exp \{-w(u)\}$ for all $u \in \mathbb{D}$. Observe that $w(1)=0$ since $g(1)=1$ and $d=-w^{\prime}(1)=1$. Invoking Lemma 2 , it follows that

$$
\operatorname{Re}(w(x)) \leq 2 \frac{1-x}{1+x},
$$

for all $x \in(-1,1)$. Hence

$$
\begin{aligned}
|g(x)| & =|\exp \{-w(x)\}|=\exp (-\operatorname{Re}\{w(x)\}) \\
& \geq \exp \left(-2 \frac{1-x}{1+x}\right),
\end{aligned}
$$

for all $x \in(-1,1)$. That is,

$$
\begin{aligned}
& \left(1-2 \log \left(\frac{1+x}{2}\right)\right)^{-1}\left|f^{\prime}\left(\frac{1-x}{2} \zeta\right)\right| \\
& \geq \exp \left(-2 \frac{1-x}{1+x}\right),
\end{aligned}
$$

for all $x \in(-1,1)$.

Making the change $r=(1-x) / 2$, we obtain that $r \in(0,1)$ and

$$
\left|f^{\prime}(r \zeta)\right| \geq \exp \left(-2 \frac{r}{1-r}\right)(1-2 \log (1-r)) .
$$

Therefore, if we consider $r=|z|$ with $z \in \mathbb{D} \neq 0$ and we take $\zeta=z /|z|$, we conclude that

$$
\begin{aligned}
\left|f^{\prime}(z)\right| & \geq \exp \left(-2 \frac{|z|}{1-|z|}\right)(1-2 \log (1-|z|)) \\
& =F^{\prime}(|z|) .
\end{aligned}
$$

This shows inequality (1).

Now, if there exists $f_{0} \in \beta_{\log }^{(\infty)}$ such that $\left|f_{0}^{\prime}\left(z_{0}\right)\right|=F^{\prime}\left(\left|z_{0}\right|\right)$ for some $z_{0} \in \mathbb{D} \backslash\{0\}$, then arguing as in the proof of inequality (1), for $\zeta_{0}=z_{0} /\left|z_{0}\right|$, the function,

$$
g_{0}(z)=\left(1-2 \log \left(\frac{1+z}{2}\right)\right)^{-1} f_{0}^{\prime}\left(\frac{1-z}{2} \zeta_{0}\right),
$$

maps $\mathbb{D}$ into $\mathbb{D} \backslash\{0\}$. Hence, there exists a holomorphic function $w$ mapping $\mathbb{D}$ into $\mathbb{H}^{+}$such that $w(1)=0,-w^{\prime}(1)=$ $1>0$, and

$$
g_{0}(z)=\exp (-w(z))
$$


for all $z \in \mathbb{D}$. In particular, for $x=1-2\left|z_{0}\right| \in(-1,1)$, we have

$$
\begin{aligned}
\exp (-\operatorname{Re} w(x)) & =\left|g_{0}(x)\right|=\frac{\mid f_{0}^{\prime}(((1-x)}{\mid 1-2 \log ((1)} \\
& =\frac{\left|f_{0}^{\prime}\left(z_{0}\right)\right|}{\left|1-2 \log \left(1-\left|z_{0}\right|\right)\right|} \\
& =\frac{F^{\prime}\left(\left|z_{0}\right|\right)}{\left|1-2 \log \left(1-\left|z_{0}\right|\right)\right|} \\
& =\exp \left(-\frac{2\left|z_{0}\right|}{1-\left|z_{0}\right|}\right) \\
& =\exp \left(-2 \frac{1-x}{1+x}\right) .
\end{aligned}
$$

Thus,

$$
\operatorname{Re} w(x)=2 \frac{1-x}{1+x}
$$

for some $x \in(-1,1)$ and, by Lemma 2 , we conclude that

$$
w(z)=2 \frac{1-z}{1+z}
$$

for all $z \in \mathbb{D}$. Therefore,

$$
\begin{array}{r}
f_{0}^{\prime}\left(\frac{1-z}{2} \zeta_{0}\right)=\left(1-2 \log \left(\frac{1+z}{2}\right)\right) g_{0}(z) \\
=\left(1-2 \log \left(\frac{1+z}{2}\right)\right) \exp \left(-2 \frac{1-z}{1+z}\right),
\end{array}
$$

for all $z \in \mathbb{D}$. Hence, changing $(1-z) / 2$ by $z$, which belongs to $D(1 / 2,1 / 2)$, and using the identity principle for holomorphic functions, we obtain that

$$
f_{0}^{\prime}\left(z \zeta_{0}\right)=F^{\prime}(z)
$$

for all $z \in \mathbb{D}$. This last relation implies that $\|F\|_{\log } \leq 1$ which is a contradiction to Proposition 7. This complete the proof of item (1).

(2) Arguing as in the proof of part (1), for $|\zeta|=1$ fixed, we set the function

$$
g(u)=\left(1-2 \log \left(\frac{1+u}{2}\right)\right)^{-1} f^{\prime}\left(\frac{1-u}{2} \zeta\right),
$$

with $u \in \mathbb{D}$. We have shown that there exists a holomorphic function $w$ such that $g(u)=\exp \{-w(u)\}$ for all $u \in \mathbb{D}$. Furthermore, $w$ satisfies the hypothesis of Julia's Lemma (Lemma 1); that is, $w$ is a holomorphic function in $\mathbb{D} \cup\{1\}$, which maps $\mathbb{D}$ into $\mathbb{U}^{+}, w(1)=0$, and $-w^{\prime}(1)=d=1$. Hence, for $r=(1-x) /(1+x)$ with $x \in[0,1)$ fixed, $w$ maps the horodisk $\bar{\Delta}(1, r)$ into the open Euclidean disk with center at $(1-x) /(1+x)$ and radius $(1-x) /(1+x)$. In particular, since $x \in \bar{\Delta}(1, r)$, we have that $w(x) \in D_{x}=D((1-x) /(1+x),(1-$ $x) /(1+x))$. Thus, Lemma 4 allows us to write

$$
\begin{aligned}
\operatorname{Re}(g(x)) & =\operatorname{Re}(\exp (-w(x))) \\
& \geq \min \left\{\operatorname{Re}(\exp (-z)): z \in D_{x}\right\} \\
& =\exp \left(-2 \frac{1-x}{1+x}\right)
\end{aligned}
$$

for all $x \in[0,1)$. This last inequality is equivalent to writing

$$
\begin{aligned}
& \operatorname{Re}\left(\left(1-2 \log \left(\frac{1+x}{2}\right)\right)^{-1} f^{\prime}\left(\frac{1-x}{2} \zeta\right)\right) \\
& \quad \geq \exp \left(-2 \frac{1-x}{1+x}\right),
\end{aligned}
$$

for all $x \in[0,1)$ and from here we have

$$
\begin{aligned}
& \operatorname{Re}\left(f^{\prime}\left(\frac{1-x}{2} \zeta\right)\right) \\
& \quad \geq \exp \left(-2 \frac{1-x}{1+x}\right)\left(1-2 \log \left(\frac{1+x}{2}\right)\right) .
\end{aligned}
$$

Making the change $r=(1-x) / 2$, we obtain $r \in(0,1 / 2]$ since $x \in[0,1)$ and also

$$
\operatorname{Re}\left(f^{\prime}(r \zeta)\right) \geq \exp \left(-2 \frac{r}{1-r}\right)(1-2 \log (1-r)) .
$$

We conclude, as before, that

$$
\operatorname{Re}\left(f^{\prime}(z)\right) \geq \exp \left(-2 \frac{|z|}{1-|z|}\right)(1-2 \log (1-|z|)),
$$

for all $|z| \leq 1 / 2$. This shows the inequality in the second part of the theorem.

Now, if there exists a function $f_{0} \in \beta_{\log }^{(\infty)}$ such that $\operatorname{Re} f^{\prime}\left(z_{0}\right)=F^{\prime}\left(\left|z_{0}\right|\right)$ for some $z_{0} \in D(0,1 / 2)$, then we can define $\zeta_{0}=z_{0} /\left|z_{0}\right|$ and the function

$$
g_{0}(z)=\left(1-2 \log \left(\frac{1+z}{2}\right)\right)^{-1} f_{0}^{\prime}\left(\frac{1-z}{2} \zeta_{0}\right),
$$

which maps $\mathbb{D}$ into $\mathbb{D} \backslash\{0\}$. Hence, as before, there exists a holomorphic function $w$ mapping $\mathbb{D}$ into $\mathbb{\boxplus}^{+}$such that $w(1)=$ $0,-w^{\prime}(1)=1>0$, and $g_{0}(z)=\exp (-w(z))$ for all $z \in \mathbb{D}$. In particular, for $x=1-2\left|z_{0}\right| \in[0,1)$, we have

$$
\begin{aligned}
\operatorname{Re} \exp (-w(x)) & =\operatorname{Re} g_{0}(x)=\frac{\operatorname{Re} f_{0}^{\prime}\left(((1-x) / 2) \zeta_{0}\right)}{1-2 \log ((1+x) / 2)} \\
& =\frac{\operatorname{Re} f_{0}^{\prime}\left(z_{0}\right)}{1-2 \log \left(1-\left|z_{0}\right|\right)} \\
& =\frac{F^{\prime}\left(\left|z_{0}\right|\right)}{1-2 \log \left(1-\left|z_{0}\right|\right)} \\
& =\exp \left(-\frac{2\left|z_{0}\right|}{1-\left|z_{0}\right|}\right) \\
& =\exp \left(-2 \frac{1-x}{1+x}\right) ;
\end{aligned}
$$

that is, $w(x)$ is the value in $D_{x}$ where $\operatorname{Re} \exp (-w(x))$ attain its minimum value, but by the proof of Lemma 4 , we know that this happens if $\operatorname{Im} w(x)=0$ and $\operatorname{Re} w(x)=-2((1-x) /(1+x))$. Now, by Lemma 2 , we conclude that $w(z)=2((1-z) /(1+z))$ for all $z \in \mathbb{D}$. As before, this last fact implies that $f_{0}^{\prime}\left(z \zeta_{0}\right)=$ $F^{\prime}(z)$ for all $z \in \mathbb{D}$ and therefore $\|F\|_{\log } \leq 1$ which is a contradiction to Proposition 7. This completes the proof of item (2). 


\section{Distortion Theorems for Complex Functions in $\mathscr{B}_{\log }$ Having Branch Points}

In this section we establish a distortion theorem for functions in the closed unit ball of $\mathscr{B}_{\log }$ having branch points and satisfying a normalized Bloch conditions. More precisely, for each $n \in \mathbb{N}$, we denote by $\beta_{\log }^{(n)}$ the class of all holomorphic functions $f \in \mathscr{B}_{\log }$ such that $f(0)=0, f^{\prime}(0)=1,\|f\|_{\log } \leq 1$ and if $f^{\prime}(b)=0$ for some $b \in \mathbb{D}$ then $f^{(k)}(b)=0$ for all $k=1,2, \ldots, n$. Clearly we have

$$
\beta_{\log }^{(\infty)}=\bigcap_{n=1}^{\infty} \beta_{\log }^{(n)}
$$

With these notations, we have the following result.

Theorem 10. For $n \in \mathbb{N}$ fixed, we set $a_{n}=\sqrt{n /(n+2)}$. Then for every $f \in \beta_{\log }^{(n)}$ we have the following:

(1) $\left|f^{\prime}(z)\right| \geq F_{n}^{\prime}(|z|)=\left(\left(a_{n}-|z|\right) /\left(a_{n}-a_{n}^{2}|z|\right)\right)^{n}(1-2 \log (1-$ $\left.\left.a_{n}|z|\right)\right)$, for all $|z| \leq a_{n}$. There is not a function $f_{0} \in \beta_{\log }^{(n)}$ such that $\left|f_{0}^{\prime}\left(z_{0}\right)\right|=F_{n}^{\prime}\left(\left|z_{0}\right|\right)$ for some $z_{0} \in \bar{D}\left(0, a_{n}\right) \backslash$ $\{0\}$.

(2) $\operatorname{Re} f^{\prime}(z) \geq F_{n}^{\prime}(|z|)=\left(\left(a_{n}-|z|\right) /\left(a_{n}-a_{n}^{2}|z|\right)\right)^{n}(1-$ $\left.2 \log \left(1-a_{n}|z|\right)\right)$, for all $|z| \leq \sqrt{n(n+2)} /(2 n+1)$. Furthermore, there is not a function $f_{0} \in \beta_{\log }^{(n)}$ such that $\operatorname{Re} f_{0}^{\prime}\left(z_{0}\right)=F_{n}^{\prime}\left(\left|z_{0}\right|\right)$ for some $z_{0} \in \bar{D}(0$, $\sqrt{n(n+2)} /(2 n+1)) \backslash\{0\}$.

Proof. (1) Let us fix $|\zeta|=1, n \in \mathbb{N}$ and $a_{n}=\sqrt{n /(n+2)}<1$. We set the function

$$
\begin{aligned}
g(u)= & \left(1-2 \log \left(1-a_{n}^{2}\right)+2 \log \left(1-a_{n}^{2} u\right)\right)^{-1} \\
& \cdot f^{\prime}\left(\frac{a_{n}-a_{n} u}{1-a_{n}^{2} u} \zeta\right)
\end{aligned}
$$

with $u \in \mathbb{D}$, where $\log (w)$ denotes the principal logarithmic of the complex number $w \in D(1,1)$. Clearly the function $g$ is holomorphic on $\mathbb{D} \cup\{1\}$ and $g(1)=1$ because $f^{\prime}(0)=1$. Also, we have

$$
\begin{aligned}
g^{\prime}(u)= & -\left(1-2 \log \left(1-a_{n}^{2}\right)+2 \log \left(1-a_{n}^{2} u\right)\right)^{-2} \\
& \cdot\left(\frac{-2 a_{n}^{2}}{1-a_{n}^{2} u}\right) f^{\prime}\left(\frac{a_{n}-a_{n} u}{1-a_{n}^{2} u} \zeta\right) \\
+ & \left(1-2 \log \left(1-a_{n}^{2}\right)+2 \log \left(1-a_{n}^{2} u\right)\right)^{-1} \\
& \cdot f^{\prime \prime}\left(\frac{a_{n}-a_{n} u}{1-a_{n}^{2} u} \zeta\right)\left[\frac{a_{n}\left(a_{n}^{2}-1\right)}{\left(1-a_{n}^{2} u\right)^{2}}\right] \zeta .
\end{aligned}
$$

And hence $g^{\prime}(1)=2 a_{n}^{2} /\left(1-a_{n}^{2}\right)=n$ since $f^{\prime}(0)=1$ and $f^{\prime \prime}(0)=0$ (by Lemma 6). Furthermore, since $f \in \beta_{\log }^{(n)}$ and $g\left(u_{0}\right)=0$ if and only if $f^{\prime}\left(\left(\left(a_{n}-a_{n} u_{0}\right) /\left(1-a_{n}^{2} u_{0}\right)\right) \zeta\right)=0$, we conclude that all the zeros of the function $g$ have multiplicity at less $n$.

On the other hand, since $\|f\|_{\log } \leq 1$ we have

$$
\begin{aligned}
& |g(u)| \leq\left|1-2 \log \left(1-a_{n}^{2}\right)+2 \log \left(1-a_{n}^{2} u\right)\right|^{-1} \\
& \cdot \log \left(\frac{e}{1-\left|\left(a_{n}-a_{n} u\right) /\left(1-a_{n}^{2} u\right)\right|^{2}}\right) \\
& \quad \leq \frac{1}{\left|\operatorname{Re}\left(1-2 \log \left(1-a_{n}^{2}\right)+2 \log \left(1-a_{n}^{2} u\right)\right)\right|} \\
& \cdot \log \left(\frac{e}{1-\left|\left(a_{n}-a_{n} u\right) /\left(1-a_{n}^{2} u\right)\right|^{2}}\right) \\
& \quad=\left[\log \left(\frac{e}{\left|\left(1-a_{n}^{2}\right) /\left(1-a_{n}^{2} u\right)\right|^{2}}\right)\right]^{-1} \\
& \cdot \log \left(\frac{e}{1-\left|\left(a_{n}-a_{n} u\right) /\left(1-a_{n}^{2} u\right)\right|^{2}}\right)<1,
\end{aligned}
$$

for all $u \in \mathbb{D}$, since

$$
\left|\frac{1-a_{n}^{2}}{1-a_{n}^{2} u}\right|^{2}<1-\left|\frac{a_{n}-a_{n} u}{1-a_{n}^{2} u}\right|^{2},
$$

for all $u \in \mathbb{D}$. Hence, we have shown that $g(\mathbb{D}) \subset \mathbb{D}$. Invoking Lemma 3, we conclude that $|g(x)| \geq x^{n}$ for all $x \in[0,1)$. Therefore, for each $x \in[0,1)$, the following estimation holds:

$$
\begin{gathered}
\left|1-2 \log \left(1-a_{n}^{2}\right)+2 \log \left(1-a_{n}^{2} x\right)\right|^{-1} \\
\cdot\left|f^{\prime}\left(\frac{a_{n}-a_{n} x}{1-a_{n}^{2} x} \zeta\right)\right| \geq x^{n} .
\end{gathered}
$$

That is,

$$
\begin{aligned}
& \left|f^{\prime}\left(\frac{a_{n}-a_{n} x}{1-a_{n}^{2} x} \zeta\right)\right| \\
& \quad \geq x^{n}\left(1-2 \log \left(1-a_{n}^{2}\right)+2 \log \left(1-a_{n}^{2} x\right)\right),
\end{aligned}
$$

for all $x \in[0,1)$ since $a_{n} \in(0,1)$.

Next, we make the change $r=\left(a_{n}-a_{n} x\right) /\left(1-a_{n}^{2} x\right)$. Then $r \in\left(0, a_{n}\right]$ since $x \in[0,1), x=\left(1 / a_{n}\right)\left(\left(a_{n}-r\right) /\left(1-a_{n} r\right)\right)$ and we can write

$$
\begin{aligned}
& \left|f^{\prime}(r \zeta)\right| \geq\left(\frac{1}{a_{n}} \frac{a_{n}-r}{1-a_{n} r}\right)^{n} \\
& \quad \cdot\left(1-2 \log \left(1-a_{n}^{2}\right)+2 \log \left(1-a_{n}^{2} \frac{1}{a_{n}} \frac{a_{n}-r}{1-a_{n} r}\right)\right) \\
& \quad=\left(\frac{1}{a_{n}} \frac{a_{n}-r}{1-a_{n} r}\right)^{n}\left(1-2 \log \left(1-a_{n} r\right)\right) .
\end{aligned}
$$


Finally, if we set $\zeta=z /|z|$ and $r=|z|$, we conclude that

$$
\begin{aligned}
\left|f^{\prime}(z)\right| & \geq\left(\frac{1}{a_{n}} \frac{a_{n}-|z|}{1-a_{n}|z|}\right)^{n}\left(1-2 \log \left(1-a_{n}|z|\right)\right) \\
& =\left(\frac{1-|z| / a_{n}}{1-a_{n}|z|}\right)^{n}\left(1-2 \log \left(1-a_{n}|z|\right)\right) \\
& =F_{n}^{\prime}(|z|)
\end{aligned}
$$

for all $|z| \in\left(0, a_{n}\right]$. This shows the inequality in part (1).

The proof of the second part is similar to part (1) of Theorem 9. If there exists a function $f_{0} \in \beta_{\log }^{(n)}$ such that $\left|f_{0}^{\prime}\left(z_{0}\right)\right|=F_{n}^{\prime}\left(\left|z_{0}\right|\right)$ for some $z_{0} \in \bar{D}\left(0, a_{n}\right) \backslash\{0\}$, then we set $\zeta=z_{0} /\left|z_{0}\right|$ and the function

$$
\begin{aligned}
g_{0}(u)= & \left(1-2 \log \left(1-a_{n}^{2}\right)+2 \log \left(1-a_{n}^{2} u\right)\right)^{-1} \\
& \cdot f_{0}^{\prime}\left(\frac{a_{n}-a_{n} u}{1-a_{n}^{2} u} \zeta\right)
\end{aligned}
$$

with $u \in \mathbb{D}$. We have showed that $g_{0}$ satisfies all the hypothesis of Lemma 3. Furthermore, choosing $x \in[0,1)$ such that

$$
\frac{a_{n}-a_{n} x}{1-a_{n}^{2} x}=\left|z_{0}\right|
$$

we obtain

$$
\begin{aligned}
& \left|g_{0}\left(\frac{a_{n}-\left|z_{0}\right|}{a_{n}-a_{n}^{2}\left|z_{0}\right|}\right)\right|=\left|g_{0}(x)\right| \\
& \quad=\left(1-2 \log \left(1-a_{n}^{2}\right)+2 \log \left(\frac{1-a_{n}^{2}}{1-a_{n}\left|z_{0}\right|}\right)\right)^{-1} \\
& \cdot\left|f_{0}^{\prime}\left(z_{0}\right)\right|=\frac{F_{n}^{\prime}\left(\left|z_{0}\right|\right)}{1-2 \log \left(1-a_{n}\left|z_{0}\right|\right)} \\
& =\left(\frac{a_{n}-\left|z_{0}\right|}{a_{n}-a_{n}^{2}\left|z_{0}\right|}\right)^{n} .
\end{aligned}
$$

By Lemma 3, we conclude that $g_{0}(z)=z^{n}$ for all $z \in \mathbb{D}$. Hence,

$$
\begin{aligned}
& f_{0}^{\prime}\left(\frac{a_{n}-a_{n} z}{1-a_{n}^{2} z} \zeta\right) \\
& \quad=\left(1-2 \log \left(1-a_{n}^{2}\right)+2 \log \left(1-a_{n}^{2} z\right)\right) z^{n},
\end{aligned}
$$

for all $z \in \mathbb{D}$. Changing $\left(a_{n}-a_{n} z\right) /\left(1-a_{n}^{2} z\right)$ by $z$, we obtain that

$$
\begin{aligned}
f_{0}^{\prime}(z \zeta) & =\left(1-2 \log \left(1-a_{n} z\right)\right)\left(\frac{a_{n}-z}{a_{n}-a_{n}^{2} z}\right)^{n} \\
& =F_{n}^{\prime}(z),
\end{aligned}
$$

for all $z \in D\left(0, a_{n}\right)$ and consequently for all $z \in \mathbb{D}$. This last equality implies that $\left\|F_{n}\right\|_{\log }=\left\|f_{0}\right\|_{\log } \leq 1$ which is a contradiction to Proposition 8.
(2) As before, for $n \in \mathbb{N}$, we set $a_{n}=\sqrt{n /(n+2)}$, we fix $|\zeta|=1$, and we define the function

$$
\begin{aligned}
g(u)= & \left(1-2 \log \left(1-a_{n}^{2}\right)+2 \log \left(1-a_{n}^{2} u\right)\right)^{-1} \\
& \cdot f^{\prime}\left(\frac{a_{n}-a_{n} u}{1-a_{n}^{2} u} \zeta\right),
\end{aligned}
$$

with $u \in \mathbb{D}$. In the first part we have shown that this function satisfies the hypothesis of Lemma 3. Hence $\operatorname{Re} g(x) \geq x^{n}$ for all $(n-1) /(n+1) \leq x<1$. Therefore

$$
\begin{aligned}
& \operatorname{Re}\left(\left(1-2 \log \left(1-a_{n}^{2}\right)+2 \log \left(1-a_{n}^{2} x\right)\right)^{-1}\right. \\
& \left.\cdot f^{\prime}\left(\frac{a_{n}-a_{n} x}{1-a_{n}^{2} x} \zeta\right)\right) \geq x^{n}
\end{aligned}
$$

and thus we have

$$
\begin{gathered}
\left(1-2 \log \left(1-a_{n}^{2}\right)+2 \log \left(1-a_{n}^{2} x\right)\right)^{-1} \\
\cdot \operatorname{Re}\left(f^{\prime}\left(\frac{a_{n}-a_{n} x}{1-a_{n}^{2} x} \zeta\right)\right) \geq x^{n},
\end{gathered}
$$

which is the same as

$$
\begin{aligned}
& \operatorname{Re}\left(f^{\prime}\left(\frac{a_{n}-a_{n} x}{1-a_{n}^{2} x} \zeta\right)\right) \\
& \geq x^{n} \log \left(\frac{e}{\left|\left(1-a_{n}^{2}\right) /\left(1-a_{n}^{2} x\right)\right|^{2}}\right) .
\end{aligned}
$$

As before, we make the change $r=\left(a_{n}-a_{n} x\right) /\left(1-a_{n}^{2} x\right)$; then $0<r \leq \sqrt{n(n+2)} /(2 n+1), x=\left(1 / a_{n}\right)\left(\left(a_{n}-r\right) /\left(1-a_{n} r\right)\right)$, and

$$
\begin{aligned}
\operatorname{Re} f^{\prime}(r \zeta) \geq\left(\frac{1}{a_{n}} \frac{a_{n}-r}{1-a_{n} r}\right)^{n} \\
\quad \cdot \log \left(\frac{e}{\left[\left(1-a_{n}^{2}\right) /\left(1-a_{n}^{2}\left(1 / a_{n}\right)\left(\left(a_{n}-r\right) /\left(1-a_{n} r\right)\right)\right)\right]^{2}}\right) \\
\quad=\left(\frac{1}{a_{n}} \frac{a_{n}-r}{1-a_{n} r}\right)^{n}\left(1-2 \log \left(1-a_{n} r\right)\right) .
\end{aligned}
$$

Setting $\zeta=z /|z|$ and $r=|z|$, we conclude that

$$
\operatorname{Re} f^{\prime}(z) \geq\left(\frac{1}{a_{n}} \frac{a_{n}-|z|}{1-a_{n}|z|}\right)^{n}\left(1-2 \log \left(1-a_{n}|z|\right)\right),
$$

for all $|z| \leq \sqrt{n(n+2)} /(2 n+1)$. This shows the inequality in part (2).

If there exists a function $f_{0} \in \beta_{\log }^{(n)}$ such that $\operatorname{Re} f_{0}^{\prime}\left(z_{0}\right)=$ $F_{n}^{\prime}\left(\left|z_{0}\right|\right)$ for some $z_{0} \in \bar{D}(0, \sqrt{n(n+2)} /(2 n+1)) \backslash\{0\}$, then we set $\zeta=z_{0} /\left|z_{0}\right|$ and the function

$$
\begin{aligned}
g_{0}(u)= & \left(1-2 \log \left(1-a_{n}^{2}\right)+2 \log \left(1-a_{n}^{2} u\right)\right)^{-1} \\
& \cdot f_{0}^{\prime}\left(\frac{a_{n}-a_{n} u}{1-a_{n}^{2} u} \zeta\right),
\end{aligned}
$$


with $u \in \mathbb{D}$. We have showed that $g_{0}$ satisfies all the hypothesis of Lemma 3. Furthermore, choosing $x \in[0,1)$ such that

$$
\frac{a_{n}-a_{n} x}{1-a_{n}^{2} x}=\left|z_{0}\right|,
$$

we obtain

$$
x=\frac{1}{a_{n}} \frac{a_{n}-\left|z_{0}\right|}{1-a_{n}\left|z_{0}\right|},
$$

and since $0<\left|z_{0}\right| \leq a_{n}((n+2) /(2 n+1))$ we can see that $(n-1) /(n+1) \leq x<1$. Furthermore,

$$
\begin{aligned}
\operatorname{Re} & g_{0}\left(\frac{a_{n}-\left|z_{0}\right|}{a_{n}-a_{n}^{2}\left|z_{0}\right|}\right)=\operatorname{Re} g_{0}(x) \\
= & \left(1-2 \log \left(1-a_{n}^{2}\right)+2 \log \left(\frac{1-a_{n}^{2}}{1-a_{n}\left|z_{0}\right|}\right)\right)^{-1} \\
& \cdot \operatorname{Re} f_{0}^{\prime}\left(z_{0}\right)=\frac{F_{n}^{\prime}\left(\left|z_{0}\right|\right)}{1-2 \log \left(1-a_{n}\left|z_{0}\right|\right)} \\
= & \left(\frac{a_{n}-\left|z_{0}\right|}{a_{n}-a_{n}^{2}\left|z_{0}\right|}\right)^{n} .
\end{aligned}
$$

By Lemma 3, we conclude that $g_{0}(z)=z^{n}$ for all $z \in \mathbb{D}$. Hence, as before, this last fact implies that $\left\|F_{n}\right\|_{\log }=\left\|f_{0}\right\|_{\log } \leq$ 1 which is a contradiction to Proposition 8 .

\section{Some Estimations for the Schlicht Radius}

In this section we present some consequences of the results obtained in Sections 4 and 5. We recall that if $f$ is a holomorphic function on $\mathbb{D}$ and $z_{0} \in \mathbb{D}, r_{s}\left(z_{0}, f\right)$ denote the radius of the largest schlicht disk on the Riemann surface $f(\mathbb{D})$ centered at $f\left(z_{0}\right)$ (a schlicht disk on $f(\mathbb{D})$ centered at $f\left(z_{0}\right)$ means that $f$ maps an open subset of $\mathbb{D}$ containing $z_{0}$ conformally onto this disk). With this notation, we have the following results.

Corollary 11. If $f \in \beta_{\log }^{(\infty)}$, then

$$
\begin{aligned}
r_{s}(0, f) & \geq \int_{0}^{1} F^{\prime}(|z|) d|z| \\
& =\int_{0}^{1} \exp \left(-\frac{2 t}{1-t}\right)(1-2 \log (1-t)) d t
\end{aligned}
$$

Proof. From the definition of $r_{s}(0, f)$, it follows the fact that there exists a simply connected domain $E \subset \mathbb{D}$ containing the zero such that $f$ maps $E$ conformally onto an Euclidean disk with center at $f(0)$ and radius $r_{s}(0, f)$. This Euclidean disk must meet the boundary of $f(\mathbb{D})$ because, in other cases, the boundary of the set $E$ is a Jordan curve in the interior of $\mathbb{D}$ and we can find an open set $W \subset \mathbb{D}$ where $f$ is univalent; hence $f(W)$ contain an Euclidean disk with center at $f(0)$ and radius greater than $r_{s}(0, f)$, which contradict with the definition of $r_{s}(0, f)$. We conclude then that there is a radial segment $\Gamma$ jointing $f(0)$ to the boundary of $f(\mathbb{D})$. Let $\gamma$ be inverse image of $\Gamma$ under $f$; then $\gamma$ joint the point 0 to the boundary of $\mathbb{D}$. Thus, from Theorem 9 , it follows that

$$
\begin{aligned}
r_{s}(0, f) & =\int_{\Gamma}|d w|=\int_{\gamma}\left|f^{\prime}(z)\right||d z| \\
& \geq \int_{0}^{1} F^{\prime}(|\gamma(t)|)\left|\gamma^{\prime}(t)\right| d t \\
& =\int_{0}^{1} F^{\prime}(|\gamma(t)|) \frac{|\gamma(t)|\left|\gamma^{\prime}(t)\right|}{|\gamma(t)|} d t \\
& \geq \int_{0}^{1} F^{\prime}(|\gamma(t)|) \frac{\gamma(t) \cdot \gamma^{\prime}(t)}{|\gamma(t)|} d t=\int_{0}^{1} F^{\prime}(\tau) d \tau \\
& =\int_{0}^{1} \exp \left(-\frac{2 \tau}{1-\tau}\right)(1-2 \log (1-\tau)) d \tau \approx 0.4104136111,
\end{aligned}
$$

where we have used Cauchy-Schwarz's inequality in the fourth line, $\gamma(t) \cdot \gamma^{\prime}(t)$ is the scalar product of $\gamma(t)$ and $\gamma^{\prime}(t)$, and we have made the change $\tau=|\gamma(t)|=\sqrt{\gamma(t) \cdot \gamma(t)}$, where $\tau \rightarrow 1^{-}$as $t \rightarrow 1^{-}$. This shows the result.

While for functions in the class $\beta_{\log }^{(n)}$ we have the following. Corollary 12. Suppose that $n \in \mathbb{N}$ is fixed. If $f \in \beta_{\log }^{(n)}$, then

$$
\begin{aligned}
r_{s}(0, f) \geq \int_{0}^{\sqrt{n /(n+2)}} F_{n}^{\prime}(t) d t \\
\quad=\int_{0}^{\sqrt{n /(n+2)}}\left(\frac{1-t / a_{n}}{1-a_{n} t}\right)^{n}\left(1-2 \log \left(1-a_{n} t\right)\right) d t .
\end{aligned}
$$

Proof. Indeed, arguing as in the proof of Corollary 11, from Theorem 10, it follows that

$$
\begin{aligned}
& r_{s}(0, f)=\int_{\Gamma}|d w|=\int_{\gamma}\left|f^{\prime}(z)\right||d z| \\
& \geq \int_{0}^{\sqrt{n /(n+2)}} F_{n}^{\prime}(\tau) d \tau \\
& =\int_{0}^{\sqrt{n /(n+2)}}\left(\frac{a_{n}-\tau}{a_{n}-a_{n}^{2} \tau}\right)^{n}\left(1-2 \log \left(1-a_{n} \tau\right)\right) d t
\end{aligned}
$$

where we have used that $\tau=|\gamma(t)| \rightarrow a_{n}$ as $t \rightarrow 1^{-}$. This shows the result.

\section{Conflicts of Interest}

The authors declare that they have no conflicts of interest regarding the publication of this paper.

\section{References}

[1] P. Koebe, "Über die Uniformisierung reeller analytischer Kurven," in Göttinger Nachrichten, pp. 177-190, 1907.

[2] L. Bieberbach, Über die Koeffizienten Derjenigen Polenzreihen, Welche Eine Schlichte Abbildung des Einheitskreises Vermitteln, S.-B. Preuss. Akad. Wiss, 1916. 
[3] A. Bloch, "Les théorèmes de M. Valiron Sur les fonctions Entières et La Théorie de l' uniformitation," Annales de la Facultédes Sciences de Toulouse, vol. 17, pp. 1-22, 1925.

[4] P. L. Duren, Univalent Functions, vol. 259 of Fundamental Principles of Mathematical Sciences, Springer, New York, NY, USA, 1983.

[5] C. Pommerenke, Boundary Behaviour of Conformal Maps, vol. 299 of Fundamental Principles of Mathematical Sciences, Springer, Berlin, Germany, 1992.

[6] X. Y. Liu and D. Minda, "Distortion theorems for Bloch functions," Transactions of the American Mathematical Society, vol. 333, no. 1, pp. 325-338, 1992.

[7] H. Yanagihara, "Sharp distortion estimate for locally schlicht Bloch functions," The Bulletin of the London Mathematical Society, vol. 26, no. 6, pp. 539-542, 1994.

[8] M. Bonk, D. Minda, and H. Yanagihara, "Distortion theorems for locally univalent Bloch functions," Journal d'Analyse Mathématique, vol. 69, pp. 73-95, 1996.

[9] M. Bonk, D. Minda, and H. Yanagihara, "Distortion theorems for Bloch functions," Pacific Journal of Mathematics, vol. 179, no. 2, pp. 241-262, 1997.

[10] I. Graham and D. Minda, "A Schwarz lemma for multivalued functions and distortion theorems for Bloch functions with branch points," Transactions of the American Mathematical Society, vol. 351, no. 12, pp. 4741-4752, 1999.

[11] T. Terada and H. Yanagihara, "Sharp distortion estimate for $p$ Bloch functions," Hiroshima Mathematical Journal, vol. 40, pp. $17-36,2010$.

[12] X. Zheng and J. Wang, "Distortion theorems and schlicht radius for p-Bloch functions," Journal of Mathematical Research with Applications, vol. 33, no. 1, pp. 45-53, 2013.

[13] L. V. Ahlfors, Conformal Invariants: Topics in Geometric Function Theory, McGraw-Hill, New York, NY, USA, 1973. 


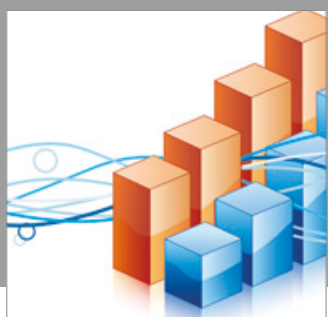

Advances in

Operations Research

vatersals

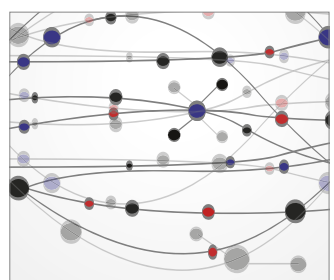

\section{The Scientific} World Journal
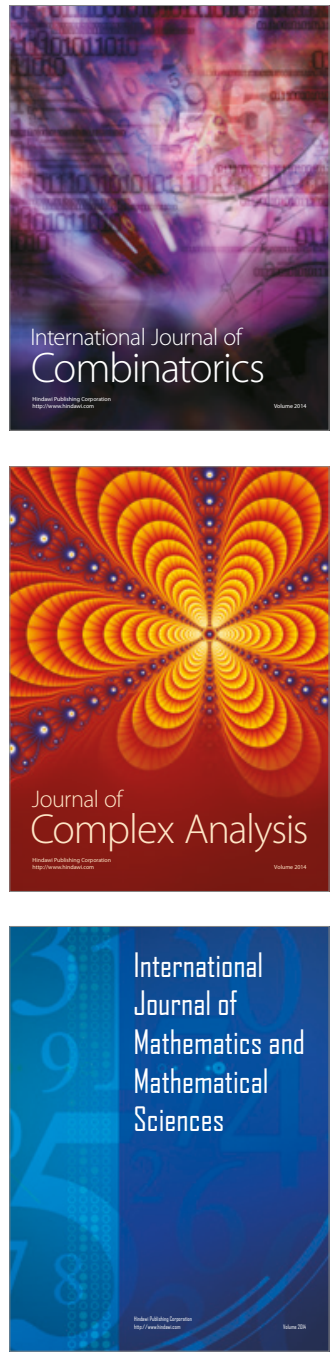
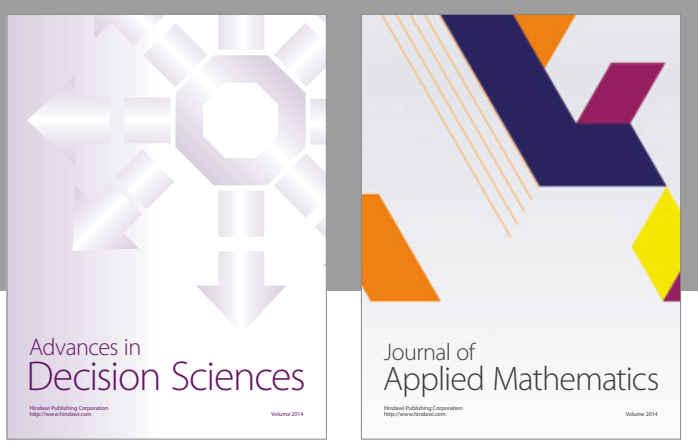

Algebra

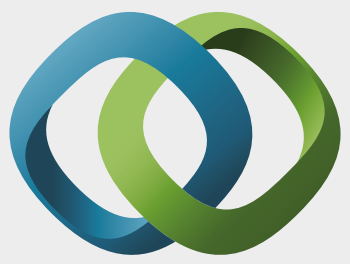

\section{Hindawi}

Submit your manuscripts at

https://www.hindawi.com
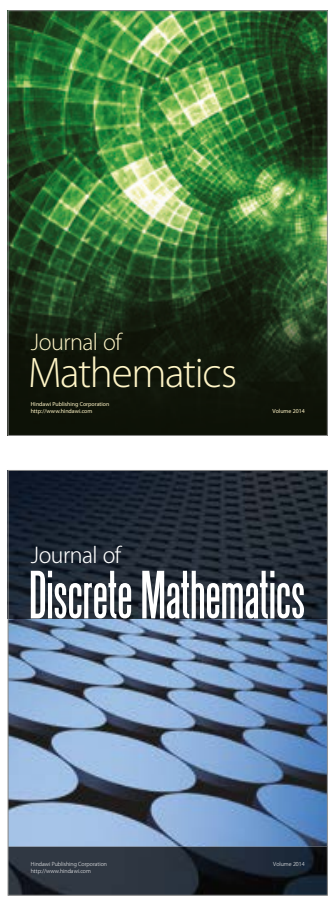

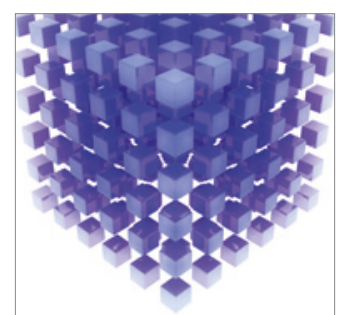

Mathematical Problems in Engineering
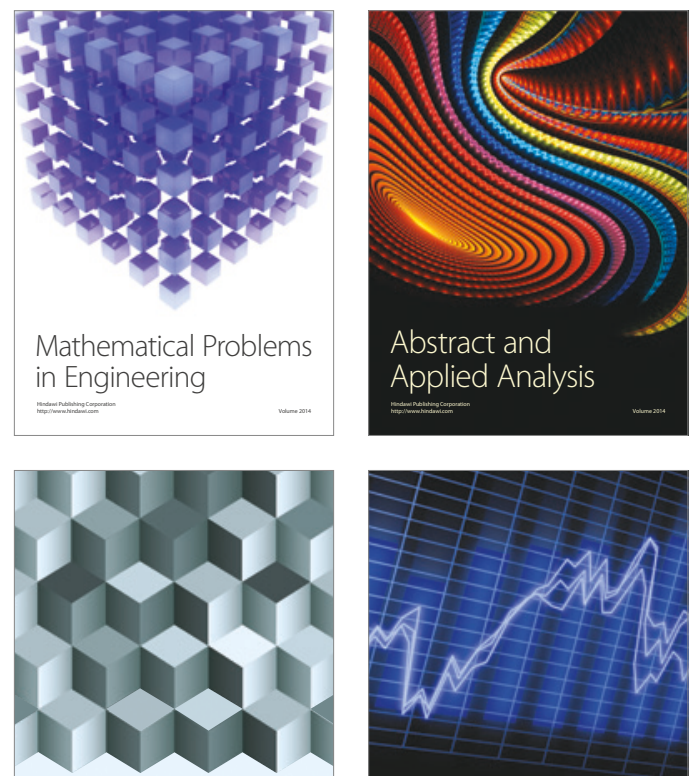

Journal of

Function Spaces

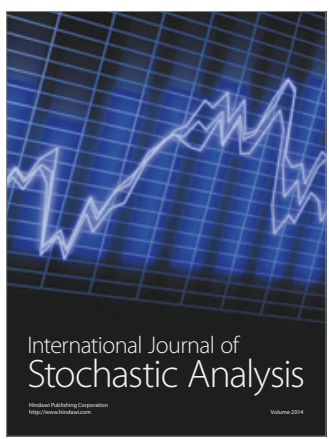

Probability and Statistics
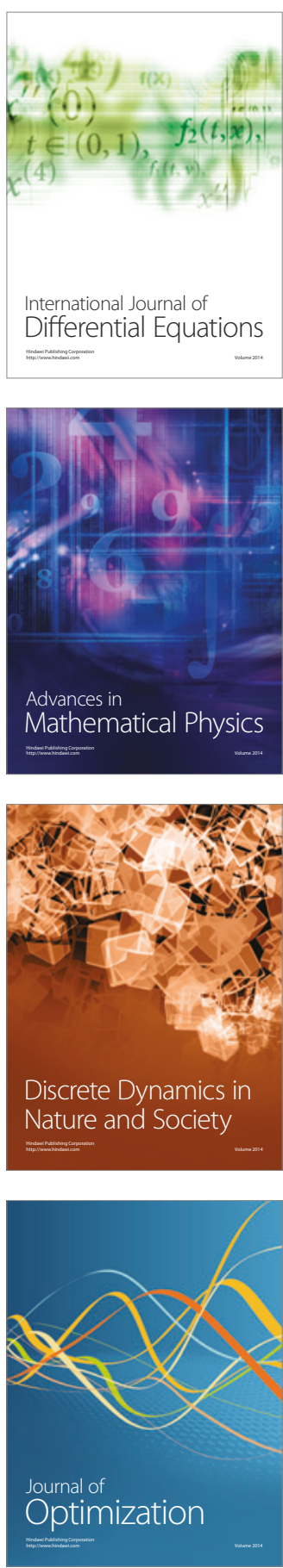